\title{
Simulation of coherent multiple imaging by means of pupil-plane filtering in optical microlithography
}

\author{
M. Erdélyi and Zs. Bor \\ Department of Optics and Quantum Electronics, JATE University H-6720 Szeged, Dóm tér 9, Hungary
}

W. L. Wilson, M. C. Smayling, and F. K. Tittel

Department of Electrical and Computer Engineering, Rice University, MS 366, Houston, Texas 77005-1892

Received October 19, 1998; revised manuscript received April 5, 1999; accepted April 12, 1999

A resolution enhancement technique for optical microlithography based on coherent multiple imaging was investigated with use of Prolith/2 (a commercial lithographic simulation tool). It was shown that a Fabry-Perot etalon placed between the mask and the projection lens of an optical stepper could be interpreted as an appropriate transmission-phase pupil-plane filter. While previous calculations were able to evaluate simple patterns (such as an on-axis contact hole), this new approach also allows the simulation of complex mask patterns. Evaluation of the point-spread function of the optical systems by means of coherent multiple imaging showed that an optimized filter is capable of increasing the resolution by $28 \%$ and the depth of focus by $150 \%$. (c) 1999 Optical Society of America [S0740-3232(99)00408-1]

OCIS codes: $110.4190,070.6110,110.4850,100.2980,110.5220,050.2230$.

\section{INTRODUCTION}

This paper discusses a new method for potentially enhancing both the depth of focus (DOF) and the resolution in projection optical lithography. This new method, which we call coherent multiple imaging (CMI), produces a series of images of the mask features, shifted in position along the optical axis, in contrast to a conventional projection process that produces only a single image at the wafer surface. Because the multiple images are added to one another coherently, their individual phase and amplitude strongly determine the final image profile.

Through proper implementation of the CMI process it is possible to control both the relative amplitude of the various images and the spacing of the images along the optical axis. A useful descriptor of a CMI system is the relative image density $\mathrm{N}$. This parameter is defined as the ratio of the depth of focus of an individual image to the spacing between the images ( $\mathrm{N}=$ DOF/image separation). When $\mathrm{N}$ is greater than 1 , images are blended together and form one continuous image. When $\mathrm{N}$ is less than 1, individual images can be observed.

Recently a CMI technique was suggested by the authors and colleagues ${ }^{1,2}$ that is based on a thin FabryPerot interferometer placed between the mask and the projection lens in a conventional photolithographic system. The relative amplitude of the individual images is controlled by the reflectance of the interferometer mirrors, while the phase and spacing of the images are determined by the mirror spacing. In Refs. 1 and 2 the spatial image distribution behind the projection lens was calculated by use of a wave-optics model. This approach was capable of evaluating the image of a simple mask pattern (such as an on-axis contact hole) but was not able to handle an arbitrary and more complex pattern, such as lines and spaces, or a contact hole array.

For evaluation of this technique for more complex and realistic mask patterns, a simulation tool such as Prolith/2 or Solid C must be employed. These tools however, are not configured in such a way that they can be immediately adapted to simulate the Fabry-Perot CMI approach. One way in which these tools can be used is to simulate each individual image by itself and then coherently add the simulations together in the proper position along the optic axis to obtain a final resultant image. ${ }^{3}$ This approach requires a modification of the original tool so that the vector image field (as opposed to the intensity distribution) can be obtained. This approach, however, is quite time consuming, as a large number of simulation runs must be executed in order to model the effect of one Fabry-Perot filter.

A more elegant and efficient approach to modeling the Fabry-Perot CMI technique is to represent the effect of the etalon with an appropriate pupil-plane filter. Enhanced image formation by means of pupil-plane filtering has been previously investigated by several authors. In particular, the super-FLEX method reported by Fukuda et al., ${ }^{4}$ introduces a pupil-plane filter that creates two images, with both the focal plane and the phase of the images shifted by an arbitrary amount. The final pattern is a superposition of these two images. Experimental and theoretical results have shown that for a contact hole pattern, super-FLEX is capable of enhancing DOF by a factor of 3 and resolution by $20 \%$. In 1992 von Bünau et al. ${ }^{5}$ used an optimized amplitude-transmission pupil-plane filter to obtain an image with an approximately constant on-axis intensity profile while maintaining most of the 
image energy within the central peak. Horiuchi et al. ${ }^{6}$ used a transmittance-adjusted pupil-plane filter to image line-space patterns. It was recently shown by the present authors (plus G. Szabo) ${ }^{7}$ that a clear-phase pupilplane filter can enhance both the DOF and the resolution of a conventional contact hole array.

\section{SIMULATION OF COHERENT MULTIPLE IMAGING WITH PUPIL-PLANE FILTERING}

Photolithographic simulation tools such as Prolith/2 and Solid C are based on F ourier optics, wherein the resultant electric-field profile is calculated as an inverse Fourier transform of the product of the Fourier transform of the mask pattern and the coherent transfer function of the optical system, $P\left(f_{x}, f_{y}\right)$. $^{8}$

$$
\begin{aligned}
E(x, y)= & \mathfrak{F}^{-1}\left\{\mathfrak{F}\{m(x, y)\} P\left(f_{x}, f_{y}\right)\right\} \\
= & \iint \mathfrak{F}\{m(x, y)\} \operatorname{circ}\left(r^{\prime} / \rho\right) \\
& \times \exp \left[2 \pi i\left(f_{x} x+f_{y} y\right)\right] d f_{x} d f_{y} .
\end{aligned}
$$

Here $m(x, y)$ is the transmittance of the mask. The spatial frequencies $f_{x}$ and $f_{y}$ are given by $x^{\prime} /(L \lambda)$ and $y^{\prime} /(L \lambda)$, where $x^{\prime}$ and $y^{\prime}$ are coordinates at the plane of the lens, $\lambda$ is the wavelength of the illuminating light, and $L$ is the distance from the mask to the lens. For a perfect, aberration-free lens, $P\left(f_{x}, f_{y}\right)$ becomes a $\operatorname{circ}\left(r^{\prime} / \rho\right)$ function that equals 1 when $r^{\prime}=\left(x^{\prime 2}+y^{\prime 2}\right)^{1 / 2}<\rho$, the radius of the lens, and equals zero elsewhere.

It is convenient to renormalize some of the variables in this expression. The numerical aperture NA is defined as $\sin \theta$, where $\theta$ is the maximum angle that a ray may have and still enter the pupil of the lens. For most optical systems, $\sin \theta \approx \rho / L=N A$. Thus if we normalize the spatial frequency by $N A / \lambda, \operatorname{circ}\left(r^{\prime} / \rho\right)$ becomes $\operatorname{circ}(r)$, where $r=\left(f_{x}^{\prime 2}+f_{y}^{\prime 2}\right)^{1 / 2}$.

It was shown in Ref. 4 that if there is a focal shift of the image, $\Delta z$, accompanied by a phase shift $\Delta \phi$, then the resultant image may be expressed as

$$
\begin{aligned}
E(x, y, z)= & \iint \mathfrak{F}\{m(x, y)\} \operatorname{circ}(r) \Delta P(\Delta \phi, \Delta z, r) \\
& \times \exp \left[2 \pi i\left(f_{x} x+f_{y} y\right)\right] d f_{x} d f_{y},
\end{aligned}
$$

with

$$
\begin{aligned}
\Delta \mathrm{P}(\Delta \phi, \Delta z, \mathrm{r})= & \exp \left[\frac{\mathrm{i} 4 \pi(\mathrm{z}-\Delta \mathrm{z})}{\mathrm{NA}^{2}}\right] \\
& \times \exp \left[2 \pi \mathrm{ir}^{2}(\mathrm{z}-\Delta \mathrm{z})\right] \exp (-\mathrm{i} \Delta \phi) .
\end{aligned}
$$

A Fabry-Perot interferometer with mirror spacing d and reflectance $\mathrm{R}$ placed between the mask and the projection lens generates a series of images of the original mask pattern whose axial spacing is $2 \mathrm{~d}$ and whose amplitude ratio is $\mathrm{R}$ (Ref. 2). Since the mirror separation is significantly smaller than the lens-mask distance, the image separation behind the lens can be approximated by
$2 \mathrm{dM}^{2}$, where $\mathrm{M}$ is the magnification of the lens. These multiple images can be accounted for by replacing $\Delta \mathrm{P}(\Delta \mathrm{z}, \Delta \phi, \mathrm{r})$ in $\mathrm{Eq}$. (2) with a new transfer function:

$$
\begin{aligned}
\Delta \mathrm{P}(\phi, \mathrm{d}, \mathrm{r})= & \mathrm{R} \exp [-\mathrm{i} \Phi(\phi, \mathrm{d}, \mathrm{r})] \\
& +\mathrm{R}^{2} \exp [-\mathrm{i} 2 \Phi(\phi, \mathrm{d}, \mathrm{r})] \\
& +\mathrm{R}^{3} \exp [-\mathrm{i} 3 \Phi(\phi, \mathrm{d}, \mathrm{r})]+\ldots,
\end{aligned}
$$

where

$$
\Phi(\phi, d, r)=\phi-2 \pi\left(\frac{2}{N A^{2}}+r^{2}\right) 2 d^{2}
$$

This relation can be written as

$$
\begin{aligned}
\Delta P(\phi, d, r)= & -R \exp [-i \Phi(\phi, d, r)] \\
& \times \frac{1}{R \exp [-i \Phi(\phi, d, r)]-1} .
\end{aligned}
$$

A Fabry-Perot interferometer can be regarded as a spatial filter that transmits certain spatial Fourier components while blocking others. Thus the similarity between a Fabry-Perot filter and a pupil-plane filter is not surprising. The calculation of a Fabry-Perot transmission function is similar to Eq. (4) but with a different phase factor.

To simulate a particular Fabry-Perot filter in a conventional simulator, the pupil radius $r$ is divided into a number of equal parts, and $\Phi(\phi, d, r)$ and hence $\Delta \mathrm{P}(\phi, \mathrm{d}, \mathrm{r})$ is determined according to $\mathrm{Eq}$. (6). This complex function is then used as a lookup table to define the pupil-plane filter function in the simulator. The conditions under which the Prolith/2 simulations were run for these tests are shown in Table 1.

To verify the validity of this approach to modeling the effect of the Fabry-Perot CMI technique, simulations were performed on the imaging of an infinitely small point source, since the results could be compared with the analytic wave-optical model reported in Ref. 2 . Two tests were evaluated for comparison. In the first test, minor changes were made to the separation of the two mirrors in the Fabry-Perot etalon. This resulted in no change to the relative image density but altered the phase difference $\phi$ between the individual images. In the second set of tests, significant changes in d were made in order to alter the observed separation between individual images. Both of these effects were evaluated theoretically and experimentally in Ref. 2, and hence a comparison of the two approaches was very straightforward.

Table 1. Input Stepper Parameters

\begin{tabular}{lc}
\hline \multicolumn{1}{c}{ Calculation Mode } & Full Scalar $^{\mathrm{a}}$ \\
\hline Numerical Aperture (NA) & 0.25 \\
Reduction $(1 / \mathrm{M})$ & 10 \\
Wavelength $(\lambda)$ & $248 \mathrm{~nm}$ \\
Spatial Coherence $(\sigma)$ & 0 \\
\hline
\end{tabular}

${ }^{a}$ Ref. 8. 

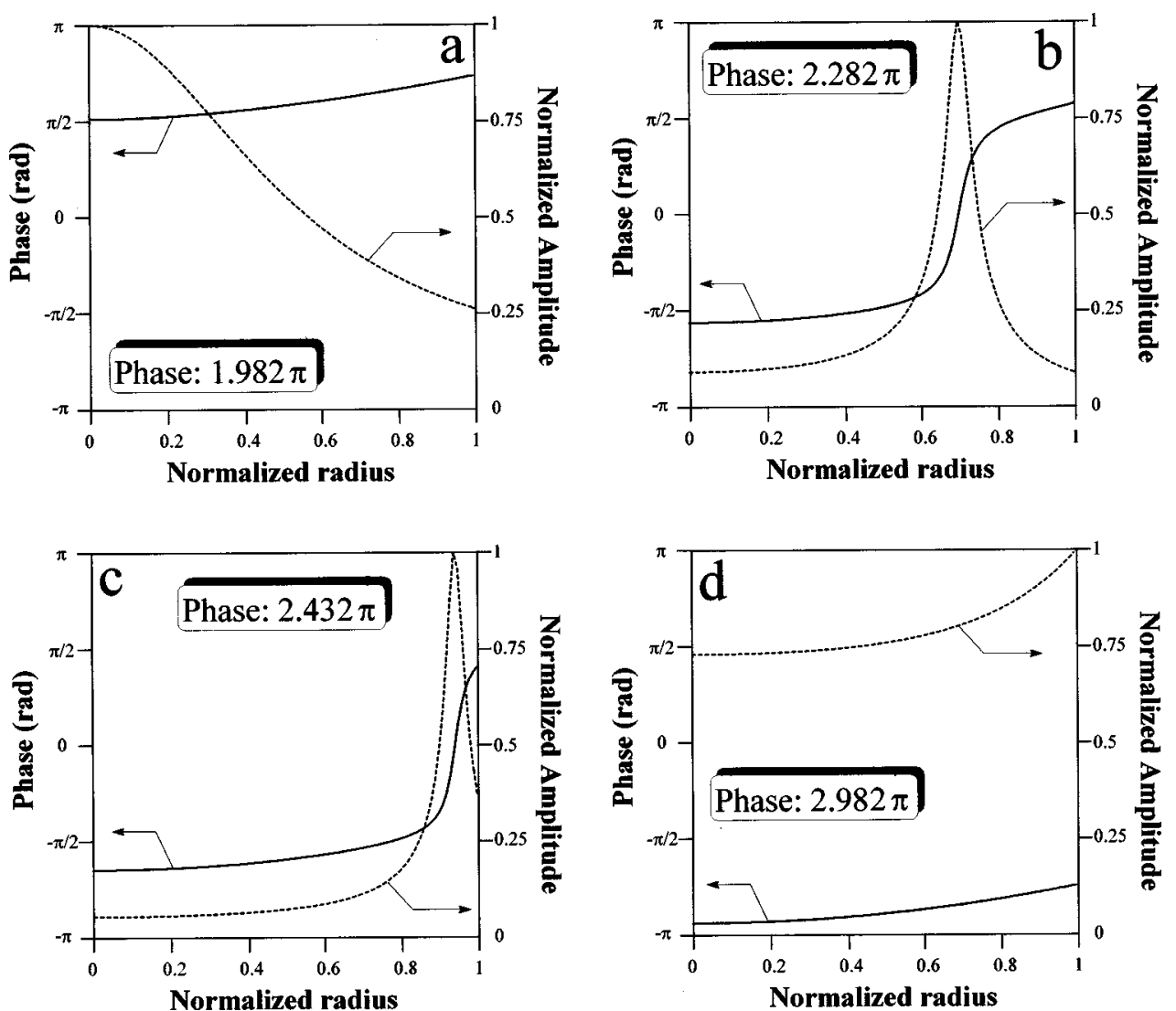

Fig. 1. Normalized amplitude (solid curves) and phase distribution (dashed curves) of the four pupil-plane filters created for a phasecontrol test. The phase values show the relative phase differences between adjacent images.

\section{PHASE CONTROL}

Significant insight into the effect of the phase setting for a given Fabry-Perot filter can be gained by plotting the amplitude and phase of $\Delta P(\phi, d, r)$, the pupil-plane transmission function, as a function of $r$. In this study four different values of $\phi$ were chosen to achieve specific transmission characteristics, as shown in Fig. 1. For purpose of discussion, these four cases have been labeled a, b, c, and $\mathrm{d}$. In all cases the relative image density $\mathrm{N}$ was the same $(N \approx 5.3)$ and only the phase $\phi$ was changed. In the case of filter a, a phase shift of $1.982 \pi$ resulted in a rather smooth transmission function, with mainly the center portion of the aperture being illuminated. Because less than the entire aperture of the lens was utilized, the resolution of the system (as determined by examination of the FWHM of the simulated point-spread function) was reduced.

Increasing $\phi$ to $2.282 \pi$, as shown for filter b, resulted in a transmission ring located approximately $3 / 4$ of the way toward the outside of the aperture. A further increase of $\phi$ to $2.432 \pi$ (filter c) sharpened the ring and moved it nearly to the outside of the aperture. This situation resulted in the most significant resolution enhancement. Setting $\phi$ to $2.982 \pi$ (filter d) moved the transmission ring outside the aperture of the lens, resulting in a reduced, more-or-less uniform, illumination of the aperture. As a result, the intensity distribution of the image was most accurately described by an Airy function.

Figure 2 depicts normalized point-spread functions of

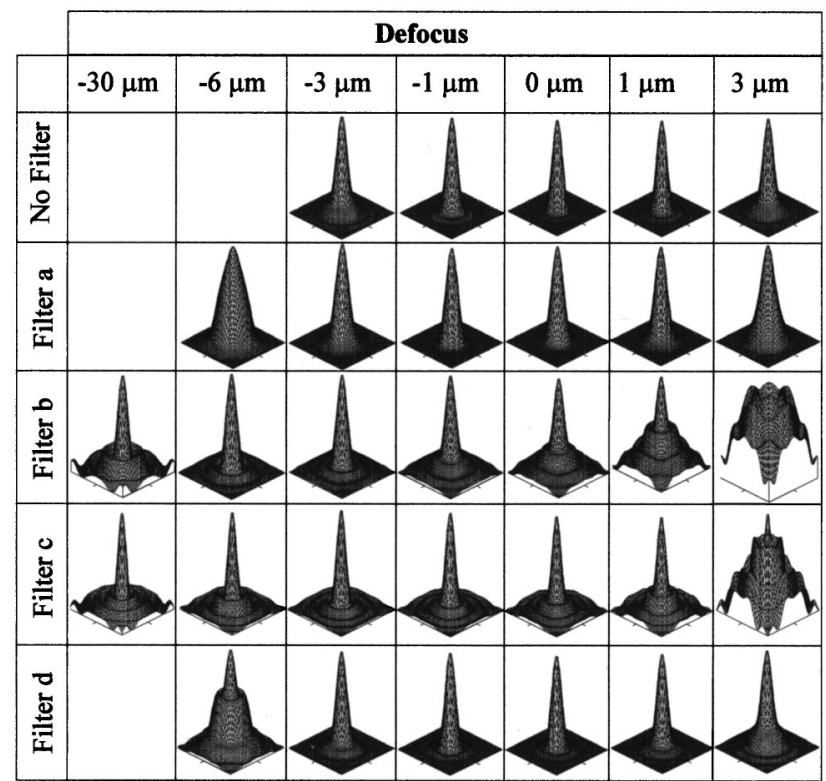

Fig. 2. Three-dimensional point-spread functions of the system without filter and with filters $a, b, c$, and d, for different defocus conditions.

the simulated optical systems for various positions along the optical axis. Results are shown for the four filters (a-d) as well as for the case of no filter at all in the system. Figure 3 shows how the Strehl ratio (the ratio of peak intensity for a point-spread function with and with- 
out a pupil-plane filter) varies along the optical axis. For the cases where the transmission through the pupil plane is more-or-less uniform (no filter, filter a, and filter $\mathrm{d}$ ), the intensity peaks are at $z=0$, the nominal focal plane for the lens. For cases $b$ and $c$, where the transmission function is sharply peaked, the maximum intensity shifts several micrometers toward the lens.

More interesting information can be gained by examining the FWHM's of the central peaks as a function of defocus for the various filters. These are shown in Fig. 4. As might be expected, the cases for no filter and for Fabry-Perot filter $d$ show nearly the same behavior. Since filter a uses less of the clear aperture of the lens, it exhibits a somewhat reduced resolution compared with $\mathrm{d}$

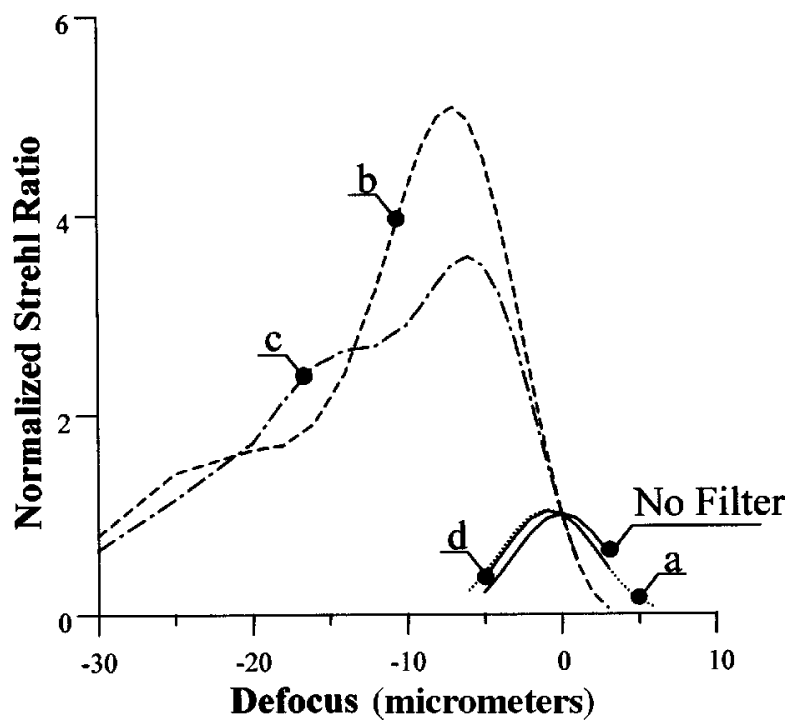

Fig. 3. Axial intensity distributions. Prolith normalizes the intensity, so that the intensity is unity in the optimum focal plane (defocus $=0$ ). Owing to constructive interference between the images, the main intensity peak is shifted toward the lens for filters $b$ and $c$. Filters $a$ and $d$ do not change the DOF.

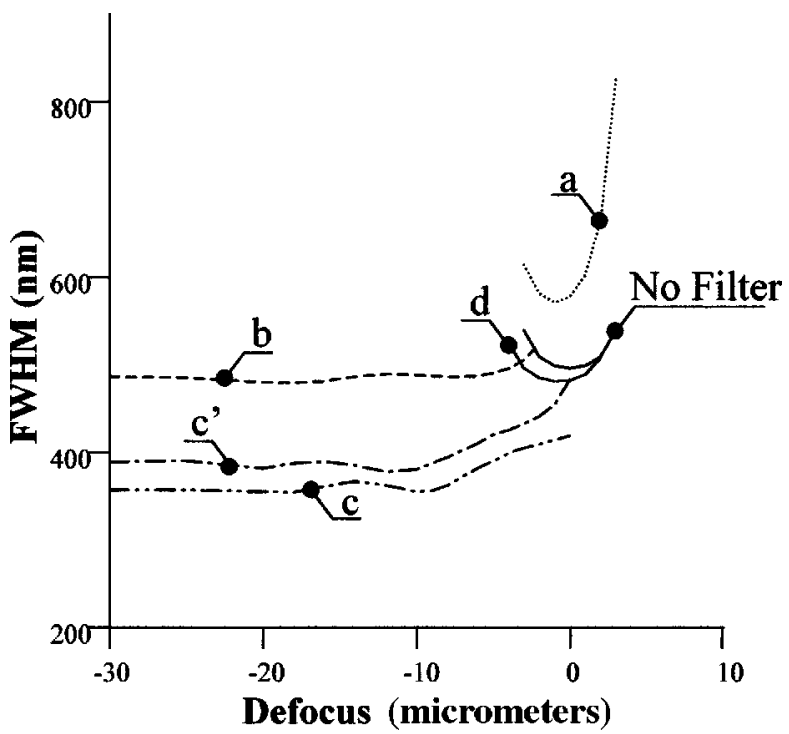

Fig. 4. FWHM of the central peaks as a function of defocus. With filters $b, c$ and $c^{\prime}$, the resolution remains constant for a large defocus range. Filter $\mathrm{d}$ does not lead to an enhancement, while filter a decreases the resolution. or the no-filter case. All three cases have nearly the same DOF. Filter $b$, which also does not utilize the full extent of the lens aperture, shows increased DOF but no significant increase in resolution. Filter c, which resulted in a narrow transmission ring at the very outside of the lens pupil, demonstrates both enhanced DOF and improved resolution. Filter c enhanced the resolution by $\sim 28 \%$ and yielded an increase in DOF of more than $150 \%$ compared with the projection lens alone. All of these re sults are in good agreement with both the theoretical and the experimental results reported in Ref. 2.

\section{IMAGE-DENSITY CONTROL}

Whereas small changes in the mirror separation of the Fabry-Perot interferometer made major changes in the phase relationship between the individual images behind the lens, these variations did not affect the image density to any significant degree. For the purpose of examining the reaction of the system to changes in image density, four new filters, $a^{\prime}, b^{\prime}, c^{\prime}$, and d', were designed. They were chosen so that each filter had a phase relationship that resulted in a transmission ring in the pupil plane located at the edge of the aperture. Filter a' had a relative image density of $\mathrm{N}=0.5$, which means that the DOF of any individual image was just one-half of the spacing between images. The filter pupil-plane transmission function with two rings for filter $a^{\prime}$ is shown in Fig. 5. The resulting image is shown in Fig. 6, where the individual images are clearly observed. The reflectance of the Fabry-Perot mirrors in this case was 0.95 , which means that the intensity ratio between individual images should be $\sim 0.9$, which is also confirmed in Fig. 6 .

Increasing $\mathrm{N}$ to 1 , as with filter $\mathrm{b}^{\prime}$, resulted in images that just overlapped. As seen in Fig. 6, the resulting intensity profile rippled with each image, but because the tails added to one another, the intensity never reached 0. Note that the average intensity fell off with defocus distance more rapidly in case $b^{\prime}$ than in case $a^{\prime}$. Filters $c^{\prime}$ and $d^{\prime}$ increased the image density even further. These filters yielded fewer oscillations in the average intensity with distance, but the overall intensity dropped off even faster than in cases $a^{\prime}$ and $b^{\prime}$.

As the relative image density is increased, the breadth of the ring in the pupil plane increases, which results in an increased intensity in the final image. At the same time, there is some degradation in the resolution enhancement as the ring gets broader. In order to arrive at final design criteria, we would require an optimization between image intensity and resolution enhancement.

\section{CONCLUSIONS}

It has been shown that a Fabry-Perot interferometer inserted between the mask and the lens in a photolithographic system is able to enhance the resolution and depth of focus simultaneously. By using an appropriate pupil-plane filter to represent the effect of the FabryPerot interferometer in a conventional lithography simulator, we have been able to verify previous theoretical predictions and to demonstrate the effectiveness of this approach on a single point source. By making small 

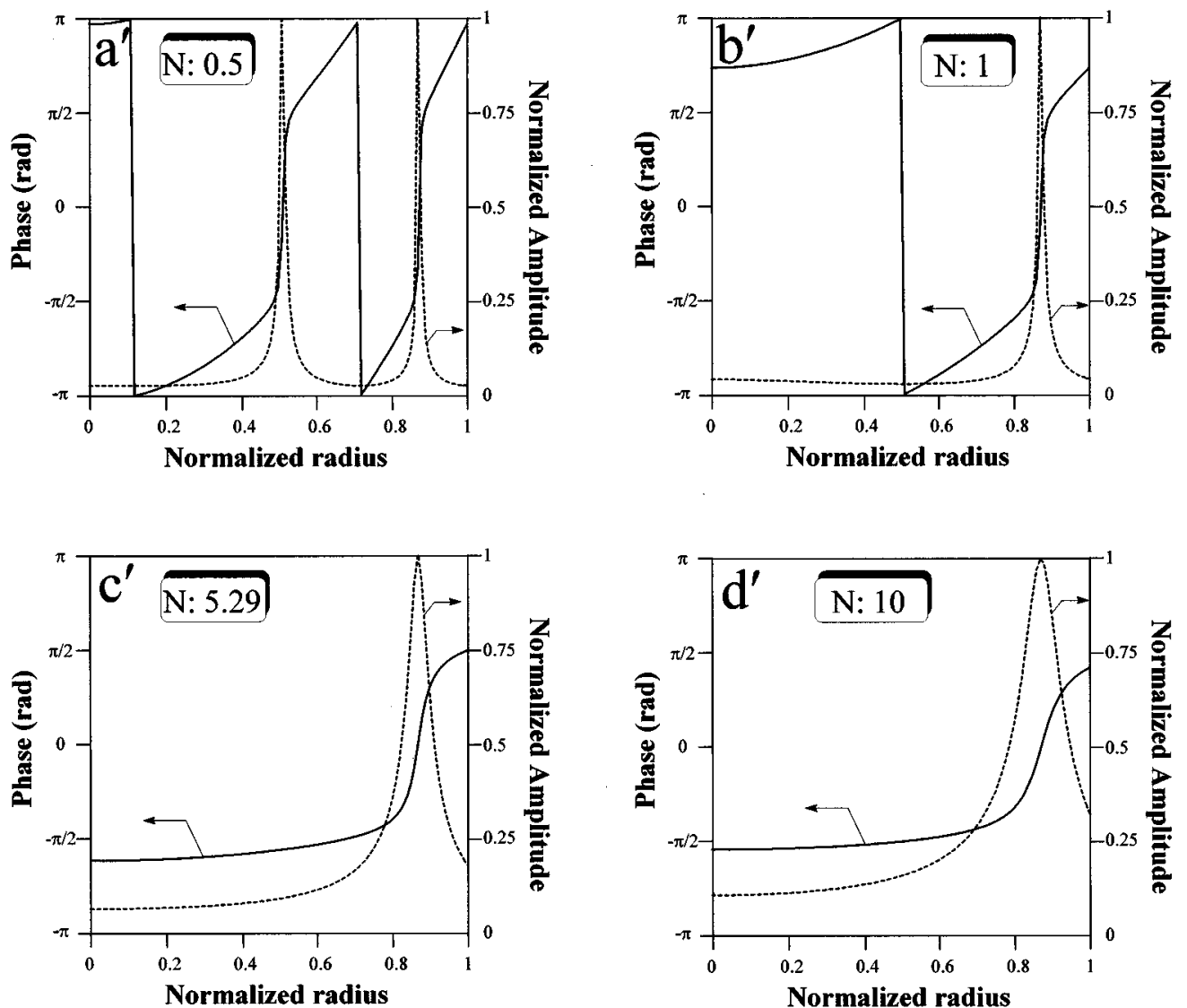

Fig. 5. Normalized amplitude (solid curves) and phase distribution (dashed curves) of four pupil-plane filters created for an imagedensity-control test. Filter a' contains two amplitude maxima, since the image density is smaller than 1 . The phase was aligned so that the transmission remains at the edge of the aperture.

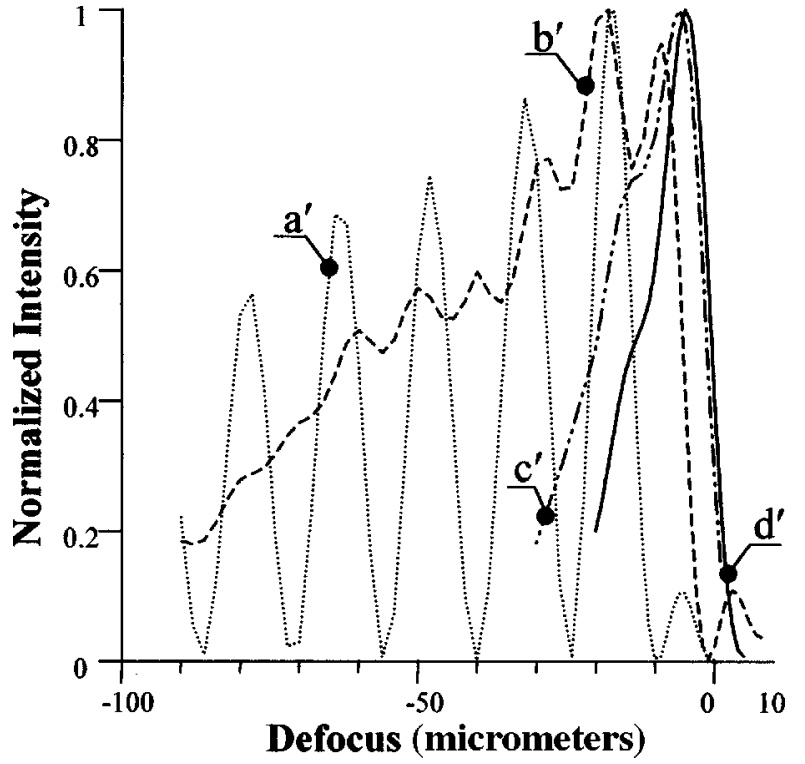

Fig. 6. Normalized axial intensity distributions. In case of filter a' the images occur separately. The images move closer together when filters $b^{\prime}, c^{\prime}$ and $d^{\prime}$ are used, and therefore oscillations disappear.

changes to the spacing $d$ between the Fabry-Perot filter mirrors, we can control the phase of the individual images that are created in the image plane. This has the effect of creating an annulus in the pupil plane. When the phase is properly adjusted, this annulus occupies the maximum aperture of the lens and results in the best resolution enhancement. By adjusting the reflectance $\mathrm{R}$ of the mirrors as well as their gross spacing, we can control the image density so as to overlap several of the images in an optimum manner and thus significantly enhance the depth of focus of the image. It is now possible to use this simulation approach to investigate the effect of a Fabry-Perot filter on more complex optical images.

\section{ACKNOWLEDGMENTS}

The authors thank Chris Mack of FI NLE Technologies for making it possible to use Prolith/2. This research was partially supported by Texas Instruments, Inc., and the OTKA Foundation of the Hungarian Academy of Sciences (T020910).

The corresponding author is F. K. Tittel: e-mail, fkt@rice.edu.

\section{REFERENCES}

1. M. Erdélyi, Z. L. Horváth, G. Szabo, Zs. Bor, F. K. Tittel, J . R. Cavallaro, and M. C. Smayling, "Generation of diffraction-free beams for application in optical microlithography," J . Vac. Sci. Technol. B 15, 287-292 (1997). 
2. Z. L. Horváth, M. Erdélyi, G. Szabo, Zs. Bor, F. K. Tittel, and J. R. Cavallaro, "Generation of nearly nondiffracting Bessel beams with a Fabry-Perot interferometer," J . Opt. Soc. Am. A 14, 3009-3013 (1997).

3. M. Erdélyi, A. Kroyen, K. Osvay, Z. Bor, W. L. Wilson, M. C. Smayling, and F. K. Tittel, "Coherent multiple imaging by means of pupil plane filtering," in Optical Microlitho graphy XII, L. van den Hove, ed., Proc. SPIE 3679, 439 (1999).

4. H. Fukuda, T. Terasawa, and S. Okazaki, "Spatial filtering for depth of focus and resolution enhancement in optical lithography," J. Vac. Sci. Technol. B 9, 3113-3116 (1991).

5. R. von Bünau, G. Owen, and R. F. W. Pease, "Depth of focus enhancement in optical lithography,"J . Vac. Sci. Technol. B 10, 3047-3054 (1992).

6. T. Horiuchi, K. Harada, S. Matsuo, Y. Takeuchi, E. Tamechika, and Y. Mimura, "Resolution enhancement by oblique illumination optical lithography using a transmittance-adjusted pupil filter,"J pn. J . Appl. Phys. 34, 1698-1708 (1995).

7. M. Erdélyi, Zs. Bor, G. Szabo, and F. K. Tittel, "Enhanced microlithography using coated objective and image duplication," in Optical Microlithography XI, L. van den Hove, ed., Proc. SPIE 3334, 579-589 (1998).

8. Chris A. Mack, Inside Prolith ${ }^{\mathrm{TM}}$; a Comprehensive Guide to Optical Lithography Simulation (FINLE Technologies, Inc., Austin, Tex., 1997). 